\title{
ALMOST OPEN MAPPINGS BETWEEN NORMED SPACES
}

\author{
ROBIN HARTE
}

ABSTRaCT. The almost open bounded linear operators between two normed spaces form an open set whose topological boundary is disjoint from the operators which are bounded below.

Introduction. Suppose $X$ and $Y$ are real or complex normed spaces, and write $\operatorname{BL}(X, Y)$ for the bounded linear operators from $X$ to $Y$. Recall that $T \in \operatorname{BL}(X, Y)$ is said to be bounded below if there is $k>0$ for which

$$
\|x\| \leqslant k\|T x\| \text { for each } x \in X,
$$

and is said to be open if there is $k>0$ for which

$$
y \in\{T x:\|x\| \leqslant k\|y\|\} \quad \text { for each } y \in Y .
$$

Evidently,

$$
T \text { bounded below } \Rightarrow \text { T one-one, } T \text { open } \Rightarrow T \text { onto, }
$$

and

$$
\begin{aligned}
T \text { invertible } & \Leftrightarrow T \text { bounded below and onto } \\
& \Leftrightarrow T \text { one-one and open. }
\end{aligned}
$$

From the point of view of invertibility, boundedness below is the bounded linear analogue of one-oneness and openness is the bounded linear analogue of ontoness. If also $S \in \mathrm{BL}(Y, Z)$ then there are implications

$$
S, T \text { bounded below } \Rightarrow S \cdot T \text { bounded below } \Rightarrow T \text { bounded below }
$$

and

$$
S, T \text { open } \Rightarrow S \cdot T \text { open } \Rightarrow S \text { open } .
$$

Algebraically, in other words, boundedness below and openness continue to resemble one-oneness and ontoness. Topologically, the operators which are bounded below form an open subset of $\operatorname{BL}(X, Y)$ : specifically if $(0.1)$ holds and if $k\left\|T^{\prime}-T\right\|$ $<1$ then for each $x \in X$,

$$
k\left\|T^{\prime} x\right\| \geqslant k\|T x\|-k\left\|T^{\prime}-T\right\|\|x\| \geqslant\left(1-k\left\|T^{\prime}-T\right\|\right)\|x\|,
$$

so that (0.1) holds with $T^{\prime}$ in place of $T$ and $k^{\prime}=k /\left(1-k\left\|T^{\prime}-T\right\|\right)$ in place of $k$. It is here that the theory suddenly begins to cloud over: we are unable to prove the analogue of $(0.7)$ for the set of open mappings, but we are instead able to prove it for

Received by the editors May 15, 1983.

1980 Mathematics Subject Classification. Primary 47A99; Secondary 46B99.

(C)1984 American Mathematical Society $0002-9939 / 84 \$ 1.00+\$ .25$ per page 
a slightly larger class of mappings, the "almost open" mappings. We make the formal definition in $\S 1$, and show that they form an open set whose topological boundary is disjoint from the set of bounded below operators. In $\$ 2$ we give some examples to show how the almost open mappings fail to completely replace the open mappings, and look at their duality theory. In $\$ 3$ we introduce a definition of "approximate invertibility", and in $\$ 4$ we look briefly at enlargements for almost open mappings.

1. The almost open mappings look only slightly more general than the open mappings:

1.1 Definition. $T \in \operatorname{BL}(X, Y)$ is said to be almost open if there is $k>0$ for which

$$
y \in \operatorname{cl}\{T x:\|x\| \leqslant k\|y\|\} \quad \text { for each } y \in Y .
$$

Readers will recognise this from its ghost-like appearance in the middle of the "open mapping theorem" [2, Lemma 5-2-2]:

$$
Y \text { complete, } T \text { onto } \Rightarrow T \text { almost open, }
$$

and

$$
X \text { complete, } T \text { almost open } \Rightarrow T \text { open. }
$$

It is elementary that

$$
T \text { open } \Rightarrow T \text { almost open } \Rightarrow T \text { dense, }
$$

and if also $S \in \operatorname{BL}(Y, Z)$ then

$S, T$ almost open $\Rightarrow S \cdot T$ almost open $\Rightarrow S$ almost open.

1.2 TheOREM. The almost open mappings form an open subset of $\operatorname{BL}(X, Y)$.

Proof. Suppose $T \in \operatorname{BL}(X, Y)$ is almost open, with $k>0$ satisfying (1.1.1), and let $T^{\prime} \in \mathrm{BL}(X, Y)$ satisfy $k\left\|T^{\prime}-T\right\|<1$ : then we claim that $T^{\prime}$ satisfies (1.1.1) with $k^{\prime}=k /(1-\delta)$ in place of $k$ whenever $k\left\|T^{\prime}-T\right\|<\delta<1$. To see this write $\delta=\varepsilon+k\left\|T^{\prime}-T\right\|$ and let $y \in Y$ be arbitrary: then there is $x_{1} \in X$ for which $\left\|y-T x_{1}\right\| \leqslant \varepsilon\|y\|$ and $\left\|x_{1}\right\| \leqslant k\|y\|$, so that also $\left\|y-T^{\prime} x_{1}\right\| \leqslant \delta\|y\|$. With $y_{2}=y-$ $T^{\prime} x_{1}$ in place of $y$, obtain $x_{2}$ in place of $x_{1}$; inductively obtain a sequence $\left(x_{n}\right)$ in $X$ for which, for each $n \in \mathbb{N}$,

$$
\left\|y-T^{\prime}\left(x_{1}+x_{2}+\cdots+x_{n}\right)\right\| \leqslant \delta^{n}\|y\| \quad \text { and }\left\|x_{n}\right\| \leqslant k \delta^{n-1}\|y\|
$$

so that

$$
\begin{gathered}
\left\|y-T^{\prime}\left(x_{1}+x_{2}+\cdots+x_{n}\right)\right\| \rightarrow 0 \quad \text { and } \\
\left\|x_{1}+x_{2}+\cdots+x_{n}\right\| \leqslant k\left(1+\delta+\cdots+\delta^{n-1}\right)\|y\| \leqslant k\|y\| /(1-\delta) .
\end{gathered}
$$

An operator which is bounded below and also the limit of a sequence of operators with dense range must be almost open:

1.3 TheOrem. If $T \in \mathrm{BL}(X, Y)$ then

$$
T \text { bounded below, }\left\|T-T_{n}\right\| \rightarrow 0, T_{n} \text { dense } \Rightarrow T \text { dense, }
$$

$$
T \text { bounded below, } T \text { dense } \Rightarrow T \text { almost open }
$$


Proof. Towards (1.3.1) suppose $y \in Y$ is arbitrary; then there is $\left(x_{n}\right)$ in $X$ with

$$
\left\|y-T_{n} x_{n}\right\| \leqslant\left\|T-T_{n}\right\| \rightarrow 0 \text { as } n \rightarrow \infty
$$

which gives, using $(0.1)$,

$$
\begin{aligned}
\left\|y-T x_{n}\right\| & \leqslant\left\|y-T_{n} x_{n}\right\|+\left\|T-T_{n}\right\|\left\|x_{n}\right\| \leqslant\left\|y-T_{n} x_{n}\right\|+k\left\|T-T_{n}\right\|\left\|T x_{n}\right\| \\
& \leqslant\left\|y-T_{n} x_{n}\right\|+k\left\|T-T_{n}\right\|\left\|T x_{n}-y\right\|+k\left\|T-T_{n}\right\|\|y\|,
\end{aligned}
$$

so that

$$
\left(1-k\left\|T-T_{n}\right\|\right)\left\|y-T x_{n}\right\| \leqslant\left\|y-T_{n} x_{n}\right\|+k\left\|T-T_{n}\right\|\|y\| \leqslant\left\|T-T_{n}\right\|(1+k\|y\|),
$$

which forces $\left\|y-T x_{n}\right\| \rightarrow 0$. Since $y$ was arbitrary, $T$ is dense.

Towards (1.3.2) suppose first that $Z$ is a dense subspace of $Y$ : we claim

$$
Y \subseteq \operatorname{cl}(Z) \Rightarrow\{y \in Y:\|y\| \leqslant 1\} \subseteq \operatorname{cl}\{z \in Z:\|z\| \leqslant 1\} .
$$

Indeed, if $y \in Y$ is arbitrary and $\left(z_{n}\right)$ in $Z$ satisfies $\left\|y-z_{n}\right\| \rightarrow 0$, define

$$
z_{n}^{\prime}=z_{n} \quad \text { if }\left\|z_{n}\right\| \leqslant\|y\| \quad \text { and } \quad z_{n}^{\prime}=\|y\| z_{n} /\left\|z_{n}\right\| \quad \text { if }\left\|z_{n}\right\|>\|y\|,
$$

so that

$$
\left\|z_{n}^{\prime}\right\| \leqslant\|y\| \text { and }\left\|y-z_{n}^{\prime}\right\| \leqslant\left\|y-z_{n}\right\|+\left|\|y\|-\left\|z_{n}\right\|\right| \leqslant 2\left\|y-z_{n}\right\| \rightarrow 0 .
$$

Applying this with $Z=T(X)$ and $k>0$ satisfying $(0.1)$ gives $\left(x_{n}\right)$ and $\left(x_{n}^{\prime}\right)$ with $z_{n}=T x_{n}$ and $z_{n}^{\prime}=T x_{n}^{\prime}$, so that $\left\|y-T x_{n}^{\prime}\right\| \rightarrow 0$ and $\left\|x_{n}^{\prime}\right\| \leqslant k\|y\|$.

1.4 THEOREM. If $T \in \mathrm{BL}(X, Y)$ is in the topological boundary of the almost open mappings then $T$ is not bounded below.

Proof. If $T$ is in the boundary of the almost open mappings then, by Theorem 1.2, $T$ is not itself almost open, while there is a sequence $\left(T_{n}\right)$ for which $\left\|T-T_{n}\right\| \rightarrow 0$ with each $T_{n}$ almost open, therefore, by (1.1.4), dense. Now if $T$ were bounded below, then by Theorem 1.3 it would be almost open, a contradiction.

2. The "dual" of Theorem 1.3 does not hold in general: it is possible for $T \in \mathrm{BL}(X, Y)$ to be one-one and almost open without being bounded below. It is also possible for $T$ to be onto without being almost open, and for $T$ to be almost open without being onto. As an abbreviation, say that $Y$ is weaker than $X$ if it is obtained by putting a different norm on the same linear space, in such a way that the identity $I: X \rightarrow Y$ is continuous, and that $Y$ is strictly weaker than $X$ if also the identity $I: Y \rightarrow X$ is not continuous.

2.1 THEOREM. If $X$ is a dense proper subspace of $Y$ and $T: X \rightarrow Y$ is the natural injection then $T \in \mathrm{BL}(X, Y)$ is almost open but not onto.

Proof. By (1.3.2) $T$ is almost open, while if $T$ were onto then $X=Y$.

For example, take $Y=C[0,1]$ in the usual supremum norm, with $X$ the subspace of polynomials, or of continuously differentiable functions.

2.2 THEOREM. If $X$ is complete, $Y$ is strictly weaker than $X$, and $T: X \rightarrow Y$ is the identity, then $T \in \mathrm{BL}(X, Y)$ is onto but not almost open. 
Proof. By (1.1.3) $T$ would be open if it were almost open, which would make the topology of $Y$ the same as that of $X$.

For example take $X=C[0,1]$ in the usual supremum norm, and give $Y$ the $L_{1}$-norm $x \rightarrow \int|x|$.

2.3 TheOREM. If $Y$ is complete and strictly weaker than $X$, and $T: X \rightarrow Y$ is the identity, then $T \in \mathrm{BL}(X, Y)$ is one-one and onto, and almost open, but not bounded below and not open.

Proof. By (1.1.2) $T$ is almost open, but if it were either bounded below or open then the topology of $Y$ would be the same as that of $X$.

For example [2, Problem 3-1-105], let $X$ be a dense proper subspace of a complete space $Z$ so that, via Hamel basis theory, $X=f^{-1}(0)$ for some discontinuous linear functional $f$ on $Z$. Choose $z_{0} \in Z$ for which $f\left(z_{0}\right)=1$ and then use the Hahn-Banach Theorem to find a continuous linear functional $g$ on $Z$ for which $g\left(z_{0}\right)=1$. Define $U: g^{-1}(0) \rightarrow X$ by setting $U(z)=z-f(z) z_{0}$ for each $z \in g^{-1}(0)$; now define the $Y$-norm on $X$ by setting $\|U(z)\|_{Y}=\|z\|_{Z}$ for each $z \in g^{-1}(0)$.

Remark that Theorem 2.1 also shows that the property (1.1.3) characterizes complete spaces: for if $X$ is not complete take $Y$ to be its completion and $T: X \rightarrow Y$ the natural injection.

In spite of the failure of the dual of Theorem 1.3, the dual of Theorem 1.4 holds. Appropriately, the proof is by duality. If $X$ is a normed linear space write $X^{\dagger}=$ $\operatorname{BL}(X, \mathbb{K})$ where $\mathbb{K}$ is the scalar field, for the dual space of $X$, and if $T \in \operatorname{BL}(X, Y)$ write $T^{\dagger}$ for the dual operator, or “adjoint", of $T$, in $\operatorname{BL}\left(Y^{\dagger}, X^{\dagger}\right)$ :

$$
T^{\dagger}(g)=g \cdot T \text { for each } g \in Y^{\dagger} .
$$

We recall, by the Hahn-Banach Theorem, that

$$
\left\|T^{\dagger}\right\|=\|T\|
$$

also by [2, Theorem 11-3-4; 1 , Theorem 1.2],

$$
T^{\dagger} \text { almost open } \Rightarrow T \text { bounded below } \Rightarrow T^{\dagger} \text { open },
$$

and

$$
T \text { almost open } \Rightarrow T^{\dagger} \text { bounded below } \Rightarrow T \text { almost open } .
$$

Indeed both implications in (2.3.3) are simple applications of the Hahn-Banach Theorem; the first implication in (2.3.4) is very elementary, while the second needs not just the Hahn-Banach Theorem but the harder "separation theorem".

From (2.3.3) and (2.3.4), using also (2.3.2), it is clear that Theorem 1.2 is equivalent to the dual result (0.7). We also obtain the dual of Theorem 1.3:

2.4 TheOREM. If $T \in \mathrm{BL}(X, Y)$ is in the topological boundary of the bounded below operators then $T$ is not almost open.

Proof. If $T$ is in the boundary of the bounded below operators then by (0.7) $T$ is not itself bounded below, which by (2.3.3) means $T^{\dagger}$ is not almost open, while there exist $\left(T_{n}\right)$ in $\operatorname{BL}(X, Y)$ which are bounded below and satisfy $\left\|T-T_{n}\right\| \rightarrow 0$. By (2.3.3) each $T_{n}^{\dagger}$ is almost open and by (2.3.2) $\left\|T^{\dagger}-T_{n}^{\dagger}\right\| \rightarrow 0$. We have therefore 
proved that $T^{\dagger}$ is in the topological boundary of the almost open operators in $\mathrm{BL}\left(Y^{\dagger}, X^{\dagger}\right)$. By Theorem 1.4 $T^{\dagger}$ is not bounded below, and then by (the elementary half of) (2.3.4) $T$ is not almost open.

3. Theorem 1.4 was sensed by pondering a familiar result in normed algebras. To present this idea suppose normed algebras $A$ and $B$ satisfy

$$
I \in A \subseteq \mathrm{BL}(X, X) \text { and } I \in B \subseteq \mathrm{BL}(Y, Y),
$$

and suppose $M$ is a linear subspace of $\operatorname{BL}(Y, X)$ for which

$$
A M \subseteq M \text { and } M B \subseteq M .
$$

Then if $T \in \operatorname{BL}(X, Y)$ satisfies

$$
T M \subseteq B \text { and } M T \subseteq A,
$$

define composition mappings $L_{T}: M \rightarrow B$ and $R_{T}: M \rightarrow A$ by setting

$$
L_{T}(S)=T S \text { and } R_{T}(S)=S T \quad \text { for each } S \in M \text {. }
$$

Say that $T \in \operatorname{BL}(X, Y)$ is left $M$-invertible iff there is $S \in M$ for which $S T=I$, and right $M$-invertible iff there is $S \in M$ for which $T S=I$; then evidently

$$
R_{T} \text { onto } \Rightarrow T \text { left } M \text {-invertible } \Rightarrow R_{T} \text { open, }
$$

and

$$
L_{T} \text { onto } \Rightarrow T \text { right } M \text {-invertible } \Rightarrow L_{T} \text { open } .
$$

3.1 Definition. $T \in \mathrm{BL}(X, Y)$ is said to be approximately left $M$-invertible if there is $\left(S_{n}\right)$ in $M$ for which

$$
\left\|I-S_{n} T\right\| \rightarrow 0 \text { as } n \rightarrow \infty
$$

and is said to be approximately right $M$-invertible if there is $\left(S_{n}\right)$ in $M$ for which

$$
\left\|I-T S_{n}\right\| \rightarrow 0 \text { as } n \rightarrow \infty \text {. }
$$

3.2 Theorem. Suppose $M \subseteq \mathrm{BL}(Y, X)$ and $T \in \mathrm{BL}(X, Y)$ satisfy (3.0.3): then

$$
\begin{aligned}
R_{T} \text { dense } & \Rightarrow T \text { approximately left } M \text {-invertible } \\
& \Rightarrow R_{T} \text { almost open, }
\end{aligned}
$$

and

$$
\begin{aligned}
L_{T} \text { dense } & \Rightarrow T \text { approximately right } M \text {-invertible } \\
& \Rightarrow L_{T} \text { almost open }
\end{aligned}
$$

Also

$$
\begin{gathered}
R_{T} \text { almost open } \Rightarrow L_{T} \text { bounded below, and } \\
L_{T} \text { almost open } \Rightarrow R_{T} \text { bounded below. }
\end{gathered}
$$

Proof. If $R_{T}$ is dense then $I \in \operatorname{cl}\{S T: S \in M\}$, giving (3.1.1). If (3.1.1) holds there is $S_{0} \in M$ for which $\left\|I-S_{0} T\right\|<1$, and if we take

$$
U=I-S_{0} T \text { and } S_{n}=\left(I+U+\cdots+U^{n}\right) S_{0}
$$

then

$$
S_{n} \in M, \quad\left\|I-S_{n} T\right\|=\left\|U^{n+1}\right\| \rightarrow 0 \quad \text { and } \quad\left\|S_{n}\right\| \leqslant\left\|S_{0}\right\| /(1-\|U\|) .
$$


More generally, if $V \in A$ is arbitrary then

(3.2.6) $\left\|V-R_{T}\left(V S_{n}\right)\right\| \rightarrow 0$ and $\left\|V S_{n}\right\| \leqslant k\|V\| \quad$ with $k=\left\|S_{0}\right\| /(1-\|U\|)$,

which means $R_{T}$ is almost open. The proof of (3.2.2) is exactly similar.

Towards the first implication of (3.2.3), if $R_{T}$ is almost open then (3.2.5) holds, and for arbitrary $S \in M$ we have

$$
\|S\| \leqslant\left\|\left(I-S_{n} T\right) S\right\|+\left\|S_{n} T S\right\| \leqslant\left\|I-S_{n} T\right\|\|S\|+k\|T S\|,
$$

giving

$$
\left(1-\left\|I-S_{n} T\right\|\right)\|S\| \leqslant k\|T S\| \text { for each } n \in \mathbb{N},
$$

which means $\|S\| \leqslant k^{\prime}\|T S\|$ whenever $k<k^{\prime}$. The argument for the second implication of (3.2.3) is exactly the same.

We refer to $T \in \operatorname{BL}(X, Y)$ for which $L_{T}$ is not bounded below as topological left M-zero divisors, and to $T \in \mathrm{BL}(X, Y)$ for which $R_{T}$ is not bounded below as topological right $M$-zero divisors. If $N \subseteq \mathrm{BL}(X, Y)$ is a linear subspace for which

$$
N A \subseteq N, \quad B N \subseteq N, \quad M N \subseteq A \text { and } N M \subseteq B,
$$

then it follows from Theorems 3.2 and 1.4 that

$$
\begin{gathered}
\partial\left\{T \in N: R_{T} \text { almost open }\right\} \pitchfork\left\{T \in N: R_{T} \text { bounded below }\right\} \\
\supseteq\left\{T \in N: L_{T} \text { almost open }\right\},
\end{gathered}
$$

and

$$
\begin{gathered}
\partial\left\{T \in N: L_{T} \text { almost open }\right\} \pitchfork\left\{T \in N: L_{T} \text { bounded below }\right\} \\
\supseteq\left\{T \in N: R_{T} \text { almost open }\right\},
\end{gathered}
$$

where $\partial K$ is the topological boundary of $K$ in $N$ and " $H \pitchfork K$ " means " $H \cap K=\varnothing$ ".

4. We conclude with a brief discussion of enlargements. If $X$ is a normed space then the quotient

$$
\mathbf{Q}(X)=l_{\infty}(X) / c_{0}(X)
$$

is called the enlargement of $X$; it contains the usual completion as a subspace. The norm of a coset has a nice expression: if $x \in l_{\infty}(X)$ then

$$
\left\|x+c_{0}(X)\right\|=\operatorname{dist}\left(x, c_{0}(X)\right)=\limsup _{n}\left\|x_{n}\right\| .
$$

If $T \in \mathrm{BL}(X, Y)$ then the enlargement of $T$ is the operator

$$
\text { (4.0.3) } \mathbf{Q}(T):\left(x_{1}, x_{2}, x_{3}, \ldots\right)+c_{0}(X) \rightarrow\left(T x_{1}, T x_{2}, T x_{3}, \ldots\right)+c_{0}(Y) \text {. }
$$

Evidently $\mathbf{Q}(T)$ is well defined, linear and bounded with $\|\mathbf{Q}(T)\|=\|T\|$. It is also by now familiar that [1, Theorem 3]

(4.0.4) $\mathbf{Q}(T)$ one-one $\Rightarrow T$ bounded below $\Rightarrow \mathbf{Q}(T)$ bounded below.

4.1 TheOREM. If $T \in \mathrm{BL}(X, Y)$ then

$$
\mathbf{Q}(T) \text { almost open } \Rightarrow T \text { almost open } \Rightarrow \mathbf{Q}(T) \text { open, }
$$

and

$\mathbf{Q}(T)$ dense $\Rightarrow T$ dense. 
Proof. If $T$ is almost open, with $k>0$ satisfying (1.1.1), and if $y \in l_{\infty}(Y)$ is arbitrary, then for each $n \in \mathbb{N}$ there is $x_{n} \in X$ with $\left\|y_{n}-T x_{n}\right\| \leqslant 1 / n$ and $\left\|x_{n}\right\| \leqslant$ $k\left\|y_{n}\right\|$; in particular, $\|x\|_{\infty} \leqslant k\|y\|_{\infty}<\infty$ so that $x \in l_{\infty}(X)$, and then

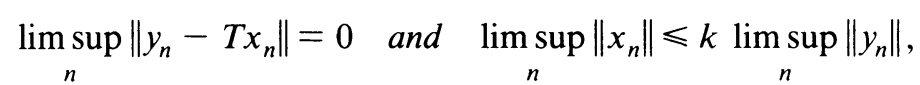

which says that $\mathbf{Q}(T)$ is open. Conversely, if $\mathbf{Q}(T)$ is almost open then for each $y \in Y$ and each $\varepsilon>0$ there is $x^{\varepsilon}=\left(x_{n}^{\varepsilon}\right)$ in $l_{\infty}(X)$ for which $\limsup _{n}\left\|x_{n}\right\| \leqslant k\|y\|$ and $\lim \sup _{n}\left\|y-T x_{n}\right\| \leqslant \varepsilon$. Thus if $0<k<k^{\prime}$ and $\varepsilon>0$ we can find $z^{\varepsilon}=x_{N}^{\varepsilon}$ for which $\left\|z^{\varepsilon}\right\| \leqslant k^{\prime}\|y\|$ and $\left\|y-T x^{\varepsilon}\right\| \leqslant 2 \varepsilon$, which says that $T$ is almost open. Part of this same argument gives the implication (4.1.2).

We have been unable to decide whether or not the implication

$$
\mathbf{Q}(T) \text { dense } \Rightarrow \text { T almost open }
$$

holds, which would be more closely analogous to (4.0.4). However, it is now clear that

4.2 TheOREM. If $T \in \mathrm{BL}(X, Y)$ then

$$
\begin{aligned}
\mathbf{Q}(T) \text { one-one and dense } & \Rightarrow T \text { bounded below and almost open } \\
& \Rightarrow \mathbf{Q}(T) \text { invertible. }
\end{aligned}
$$

Proof. The second implication is clear: if $T$ is bounded below and almost open then (4.0.4) and (4.1.1) say that $Q(T)$ is bounded below and open, therefore invertible. If $\mathrm{Q}(T)$ is one-one and dense then (4.0.4) and (4.1.2) say that $T$ is bounded below and dense, and (1.3.2) says that $T$ is bounded below and almost open.

\section{REFERENCES}

1. R. E. Harte, Berberian-Quigley and the ghost of a spectral mapping theorem, Proc. Roy. Irish Acad. Sect. A 78 (1978), 63-68.

2. A. Wilansky, Modern methods in topological vector spaces, McGraw-Hill, New York, 1978.

Department of Mathematics, University College, Cork, Ireland 